\title{
Selective sparing of goblet cells and Paneth cells in the intestine of methotrexate-treated rats
}

\author{
MELISSA VERBURG,${ }^{1}$ INGRID B. RENES,${ }^{1}$ HELEN P. MEIJER,${ }^{1}$ JAN A. J. M. TAMINIAU, ${ }^{2}$ \\ HANS A. BÜLLER, ${ }^{1}$ ALEXANDRA W. C. EINERHAND, ${ }^{1}$ AND JAN DEKKER ${ }^{1}$ \\ ${ }^{1}$ Laboratory of Pediatric Gastroenterology and Nutrition, Erasmus University and Sophia \\ Children's Hospital, 3015GE Rotterdam; and ${ }^{2}$ Department of Pediatrics, Academic Medical Center, \\ 1105AZ Amsterdam, The Netherlands
}

Received 10 January 2000; accepted in final form 19 May 2000

\begin{abstract}
Verburg, Melissa, Ingrid B. Renes, Helen P. Meijer, Jan A. J. M. Taminiau, Hans A. Büller, Alexandra W. C. Einerhand, and Jan Dekker. Selective sparing of goblet cells and Paneth cells in the intestine of methotrexatetreated rats. Am J Physiol Gastrointest Liver Physiol 279: G1037-G1047, 2000.-Proliferation, differentiation, and cell death were studied in small intestinal and colonic epithelia of rats after treatment with methotrexate. Days 1-2 after treatment were characterized by decreased proliferation, increased apoptosis, and decreased numbers and depths of small intestinal crypts in a proximal-to-distal decreasing gradient along the small intestine. The remaining crypt epithelium appeared flattened, except for Paneth cells, in which lysozyme protein and mRNA expression was increased. Regeneration through increased proliferation during days 3-4 coincided with villus atrophy, showing decreased numbers of villus enterocytes and decreased expression of the enterocyte-specific genes sucrase-isomaltase and carbamoyl phosphate synthase I. Remarkably, goblet cells were spared at villus tips and remained functional, displaying Muc2 and trefoil factor 3 expression. On days 8-10, all parameters had returned to normal in the whole small intestine. No methotrexate-induced changes were seen in epithelial morphology, proliferation, apoptosis, Muc2, and TFF3 immunostaining in the colon. The observed small intestinal sparing of Paneth cells and goblet cells following exposure to methotrexate is likely to contribute to epithelial defense during increased vulnerability of the intestinal epithelium.
\end{abstract}

villus atrophy; chemotherapy; gene expression; damage and regeneration

THE INTESTINAL EPITHELIUM forms a dynamic system of continuous proliferation, differentiation, and cell death. In the crypt compartment, stem cells and proliferating cells produce new epithelial cells. During migration from the proliferative compartment toward the crypt base, villus tips (small intestine), or surface epithelium (colon), epithelial cells differentiate into distinct cell types that can be identified using morphological criteria and through expression of specific genes. At villus tips and at the surface epithelium, cells

\footnotetext{
Address for reprint requests and other correspondence: J. Dekker, Laboratory of Pediatrics, Rm. Ee1571A, Erasmus Univ., Dr Molewaterplein 50, 3015GE Rotterdam, The Netherlands (E-mail: dekker@kgk.fgg.eur.nl).
}

are shed into the lumen or become phagocytosed after apoptosis (7).

Enterocytes form the largest population of epithelial cells. They express highly selective enzymes and transporter proteins that regulate nutrient uptake and fluid housekeeping in a well-orchestrated manner. Specific differences exist in the functions of enterocytes along the proximal-to-distal axis of the intestine, which are reflected in the regulated expression of region-specific genes (28). The vulnerable epithelium is protected against mechanical stress, luminal substances, and pathological microorganisms through the secretion of mucus. The mucus barrier consists mainly of mucins, which are large glycoproteins that are secreted by goblet cells $(25,27)$. The relative numbers of goblet cells increase along the proximal-to-distal axis, constituting only a few percent in the duodenum but up to $\sim 20 \%$ of the cells in the colon. Paneth cells, only present in the small intestine, are localized at the very base of the crypts and secrete antimicrobial polypeptides such as lysozyme and growth factors into the lumen. In contrast to the enterocytes and goblet cells, which have a lifespan of only 3 days in the rat, the Paneth cells have a slower turnover, resulting in a lifespan of $\sim 3$ wk (2). Another small group of slowly renewing cells is formed by the enteroendocrine cells, which specialize in the primarily mucosal secretion of hormonal peptides (3). Collectively, the epithelial cells maintain a high barrier function, principally through the presence of a tight junctional network between their plasma membranes, to separate the luminal content from the body's interior (29).

The use of the cytostatic drug methotrexate (MTX) in anticancer treatments may severely impair intestinal epithelial function and therefore constitutes a doselimiting factor in treatment schedules (20). Like other chemotherapeutics, it induces diarrhea and anorexia, accompanied by malabsorption, malnutrition, and dehydration. Research has been performed on the effects of cytostatic drugs, including MTX, on the epithelium of the small intestine in animals. The use of animal

\footnotetext{
The costs of publication of this article were defrayed in part by the payment of page charges. The article must therefore be hereby marked "advertisement" in accordance with 18 U.S.C. Section 1734 solely to indicate this fact.
} 
experiments allows the analysis of the effects of a specific drug over time in all regions of the intestine, which would be impossible to perform in humans. Previously, it was shown in rats that MTX inhibited intestinal epithelial proliferation, which was followed by villus atrophy in the rat small intestine (23). Furthermore, during damage the levels of glycohydrolase enzyme activities strongly decreased in rat jejunum (23), suggesting enterocyte malfunction. In other studies using the cytostatic drug 5-fluorouracil, expression of the aminopeptidase transporter 1 appeared resistant to similar mucosal injury in rat ileum (24). In each study, the epithelium was able to fully regenerate. During regeneration, the epithelial cells increased in number and the enterocytes regained their degradative and absorptive functions. In several studies, the prophylactic or therapeutic administration of particular growth factors, vitamins, or inhibitors of apoptosis could enhance the regenerative capacities of the epithelium in vivo $(4,8,9,11,13,18)$.

Despite numerous investigations, very little is known about the fate of the individual epithelial cell types after exposure to cytostatic drugs. Also, little attention was paid to the response of the large intestine to MTX or other cytostatics. Knowledge of cell type-specific and region-specific variation in MTX sensitivity is still lacking, which is essential for the development of oral treatments in humans that could maintain or enhance intestinal epithelial cell functions during and after exposure to cytostatic drugs. In this study, we investigated in rats the mechanism of intestinal epithelial damage and regeneration in all regions of the small intestine and in the proximal colon using specific (immuno-) histochemical stainings for proliferating cells, enterocytes, goblet cells, Paneth cells, and apoptotic cells. Furthermore, we quantified the expression of cell type-specific differentiation markers at the mRNA level. This will give us insight into the functional capacities of the epithelium during each phase of MTX-induced damage and subsequent regeneration.

\section{MATERIALS AND METHODS}

Unless otherwise indicated, chemicals and enzymes were obtained from the following manufacturers: Merck (Darmstadt, Germany); Sigma (St. Louis, MO); BDH (Poole, Dorset, UK); Boehringer Mannheim (Mannheim, Germany); Vector Laboratories (Burlingame, CA); DAKO (Glostrup, Denmark); Amersham (Little Chalfont, UK); and GIBCO BRL (Gaithersburg, MD).

Animals. Male Wag/Rij rats (Broekman, Utrecht, The Netherlands), aged 6 wk (120-140 g), were kept in conventional cages (3 rats/cage) in a specific pathogen-free environment under controlled humidity and a 12:12-h light/dark cycle (light 7 AM-7 PM) with free access to defined semisynthetic chow ( $1 \mathrm{mg} / \mathrm{kg}$ folic acid; Hope Farms, Woerden, The Netherlands) and water. The dosage schedule of MTX treatment leading to intestinal damage and regeneration without causing death was obtained from pilot studies. At 10 AM (day -1), $20 \mathrm{mg} / \mathrm{kg}$ body wt MTX (Ledertrexate SP Forte; Cyanamid Benelux, Etten-Leur, The Netherlands) was injected intravenously under light anesthesia. Twenty-four hours later (day 0 ), a second injection of $10 \mathrm{mg} / \mathrm{kg}$ body wt MTX was given. Control animals received equivalent volumes of $0.9 \%$ $\mathrm{NaCl}$ solution both times. During the experiment, food intake, body weight, diarrhea, and malaise were recorded daily. At days 1,2, 3, 4, 5, 6, 8, and 10, the MTX-treated rats were killed by decapitation. Control animals were killed at day 8. To analyze epithelial proliferation, $50 \mathrm{mg} / \mathrm{kg}$ body wt bromodeoxyuridine (BrdU; Sigma), dissolved in sterile PBS ( $\mathrm{pH}$ 7.4) was injected intraperitoneally $24 \mathrm{~h}$ before decapitation. Rats that were killed at day 1 were injected with BrdU $17 \mathrm{~h}$ before decapitation. Segments of duodenum (proximal 3 $\mathrm{cm}$ of the small intestine), jejunum (anatomic middle of the small intestine), ileum (distal $3 \mathrm{~cm}$ of the small intestine), and proximal colon (proximal $3 \mathrm{~cm}$ of the colon) were rinsed in PBS, fixed in $4 \%$ formaldehyde (Merck) dissolved in PBS, dehydrated, and embedded in Paraplast Plus (Sherwood Medical, Den Bosch, The Netherlands) according to standard procedures for (immuno-) histochemistry. In addition, small segments of the jejunum $(0.5 \mathrm{~cm})$ of each animal were frozen in liquid nitrogen and further stored at $-80^{\circ} \mathrm{C}$ for RNA isolation.

Morphology. Epithelial morphology and apoptosis were analyzed on 6 - $\mu \mathrm{m}$-thick tissue sections after standard histochemical staining methods using either alcian blue (BDH) and nuclear fast red (Merck) or hematoxylin (Vector Laboratories) and eosin (Merck) (HE). Crypt depth and villus height were measured manually in well-orientated sections (5 measurements $\cdot$ region ${ }^{-1} \cdot$ rat $^{-1}$ ) using a micrometer (Nikon, Bunnik, The Netherlands) mounted in an Eclipse E800 Microscope (Nikon, Japan).

Immunohistochemistry. Proliferation and antigen expression were detected by immunohistochemistry. Tissue sections were deparaffinized with xylene (Merck), rehydrated in graded ethanol solutions, and treated with $1.5 \%$ ( $\mathrm{vol} / \mathrm{vol}$ ) $\mathrm{H}_{2} \mathrm{O}_{2}$ (Merck) in PBS for $30 \mathrm{~min}$ at room temperature to remove endogenous peroxidase activity. Antigen unmasking was carried out by heating the sections for $10 \mathrm{~min}$ in $0.01 \mathrm{M}$ sodium citrate ( $\mathrm{pH} 6.0$; Merck) at $100^{\circ} \mathrm{C}$. For the anti-BrdU antibodies, all sections were incubated using $20 \mu \mathrm{g} / \mathrm{ml}$ proteinase $\mathrm{K}$ (Boehringer) in PBS for $7.5 \mathrm{~min}$ and colonic sections were additionally treated with $2 \mathrm{~N} \mathrm{HCl}$ (Merck) for 90 min before incubation with the protease. Nonspecific protein binding was blocked using a buffer containing $10 \mathrm{mM}$ Tris (Merck), 5 mM EDTA (Merck), $0.15 \mathrm{M} \mathrm{NaCl}$ (Merck), 0.25\% gelatin (Sigma), and 0.05\% Tween 20 (Merck), pH 8.0. All sections were incubated overnight $\left(4^{\circ} \mathrm{C}\right)$ using the primary mouse monoclonal antibodies anti-BrdU (1:100-400; Boehringer), anti-human proliferating cell nuclear antigen (PCNA, 1:2,500; Boehringer), anti-human Muc2 (1:100; see Ref. 26) or the rabbit polyclonal antibodies anti-human lysozyme (1:50; DAKO), anti-calf alkaline phosphatase (AP, 1:1,500; DAKO), anti-rat carbamoylphosphate synthase I (CPSI, 1:5,000; see Ref. 5), anti-rat sucrase-isomaltase (SI, 1:6,000; see Ref. 32), anti-rat trefoil factor 3 (TFF3, 1:6,000) or anti-rat carbonic anhydrase I (CAI, 1:6,000). Immunodetection was performed using a Vectastain elite ABC kit, peroxidase-conjugated (Vector Laboratories) and $0.5 \mathrm{mg} / \mathrm{ml} \quad 3,3^{\prime}$-diaminobenzidine (Sigma) in imidazole buffer containing $30 \mathrm{mM}$ imidazole (Merck) and $1 \mathrm{mM}$ EDTA (Merck), pH 7.0. Sections were counterstained using ready-to-use hematoxylin mounted in Entellan (Merck) and analyzed under an Eclipse E800 Microscope. To investigate whether the observed sparing of goblet cells during villus atrophy was statistically significant, Muc2-positive cells were counted in villi of the jejunum until day 4 after MTX. Ten crypt-villus axes were chosen per animal in well-orientated sections. The number and ratio of Muc2-positive cells in the lower and upper halves of the villi of control and MTX-treated rats were compared using the 
Mann-Whitney $U$-test. Similarly, the number of lysozymepositive cells were counted in 10 jejunal crypts/animal until day 4 to investigate the MTX resistance of Paneth cells.

Histochemistry. Differentiated enterocytes expressing AP activity were localized on deparaffinized, rehydrated tissue sections in an one-step assay using $50 \mu$ l nitroblue tetrazolium solution (Vector Laboratories) and $37.5 \mu \mathrm{l}$ bromochloroindolyl phosphate solution (Vector Laboratories) in 10 $\mathrm{ml}$ Tris ( $\mathrm{pH}$ 9.5) as described by the manufacturer. After a 12-min incubation at room temperature, the reaction was stopped in distilled water. After mounting in Entellan, brush border staining was analyzed under an Eclipse E800 microscope.

Terminal transferase deoxyuridine nick-end labeling. For the detection of apoptotic cells, the terminal transferase deoxyuridine nick-end labeling (TUNEL) method was used as described previously, with slight modifications (17). Briefly, following deparaffinization and rehydration, sections were incubated for $7.5 \mathrm{~min}$ with $20 \mu \mathrm{g} / \mathrm{ml}$ proteinase $\mathrm{K}$ at room temperature. After rinsing in PBS and removal of endogenous peroxidase activity for $20 \mathrm{~min}$ using $1.5 \%$ (vol/ vol) $\mathrm{H}_{2} \mathrm{O}_{2}$ in PBS, sections were preincubated in $25 \mathrm{mM}$ Tris, $\mathrm{pH} 6.6,200 \mathrm{mM}$ potassium cacodylate, $0.2 \mathrm{mM}$ EDTA, and $0.25 \mathrm{mg} / \mathrm{ml}$ BSA for $10 \mathrm{~min}$. Subsequently, new buffer was added, including $1 \mathrm{mM}$ cobalt chloride, $0.4 \mathrm{pM}$ digoxigenin11-deoxyuridine triphosphate (Boehringer), and $25 \mathrm{U} / \mathrm{ml}$ terminal transferase (Boehringer) for a 90-min incubation at $37^{\circ} \mathrm{C}$. Negative controls were included by omitting terminal transferase. Positive controls were incubated in $0.5 \mathrm{U} / \mu \mathrm{l}$ DNAse (Boehringer)-containing buffer, including $50 \mathrm{mM}$ Tris (pH 9.2), $14 \mathrm{mM}$ ammonium sulfate (Merck), 20\% (vol/vol) dimethyl sulfoxide (Merck), 0.3\% (vol/vol) Tween 20, $50 \mathrm{mM}$ $\mathrm{MgCl}_{2}$ (Boehringer), and $100 \mathrm{mM} \mathrm{CaCl}{ }_{2}$ (Boehringer), before incubation with the terminal transferase. The reaction was stopped for $15 \mathrm{~min}$ by using $300 \mathrm{mM} \mathrm{NaCl}$ and $30 \mathrm{mM}$ sodium citrate and was subsequently blocked for 10 min with $2 \%$ BSA in PBS. After rinsing in PBS, the sections were incubated overnight at $4^{\circ} \mathrm{C}$ using peroxidase-conjugated $\mathrm{Fab}$ fragments (1:2,000; Boehringer) in PBS. Binding of Fab frag-

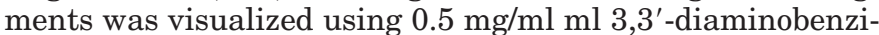
dine dissolved in imidazole buffer. Serial sections were stained using HE for comparison of TUNEL reaction with the known morphological criteria (nuclear shrinkage, apoptotic bodies) (7).

Northern blots. Total RNA was isolated from frozen jejunal segments $(0.5 \mathrm{~cm})$ using TRIzol reagent (GIBCO). Cell typespecific mRNA expression was quantified on both Northern and Northern spot blots. For the detection of Muc2 and CPSI mRNA, $2 \mu \mathrm{g}$ total RNA was spotted directly onto nylon membranes (Hybond- ${ }^{+}$, Amersham) using a Bio-Rad spot blot device (Bio-Rad, Hercules, CA). To detect SI, TFF3, and lysozyme mRNA, $10 \mu \mathrm{g}$ of total RNA was separated on $1-1.2 \%$ agarose gels (Boehringer) containing 3.5\% glyoxal (Merck) before blotting. All blots were hybridized using specific ${ }^{32}$ P-labeled cDNA probes. Hybridization signals were quantified using a PhosphorImager (Molecular Dynamics) after 1-3 days exposure time. The following probes were used: A 827-bp EcoR I fragment of rat SI mRNA (12), a 1.9-kb EcoR I fragment of rat CPSI mRNA (19), $1 \mathrm{~kb}$ of rat lysozyme mRNA (33), and $438 \mathrm{bp}$ of rat TFF3 mRNA (22). For the construction of a rat Muc2 probe, RT-PCR was performed on colonic RNA using the primers ACCTGTCGACTGGTAGAGGAGATTACCCCC and GCTCTAGATCACATGTGGTCAGGTTGC, based on the rat Muc2 coding sequence (31). Rat Muc2 (bp 506-1560, $1.1 \mathrm{~kb}$ ) was amplified and cloned in the $\mathrm{Sal}$ I-Xba I sites of pBluescript SK+ (Promega, Madison, WI). Hybridized signals were corrected for glyceraldehyde-3- phosphate dehydrogenase (GAPDH) mRNA expression to correct for the amount of loading by using $1.4 \mathrm{~kb}$ GAPDH mRNA as a probe.

Statistical analysis. Statistical analysis was performed on crypt depth, villus height, goblet cell and Paneth cell numbers, and mRNA expression using the Mann-Whitney $U$-test. Due to time-dependent intraanimal variations, data from two consecutive time points were pooled. A $P$ value of $<0.05$ was considered statistically significant.

\section{RESULTS}

Clinical symptoms. The intake of food and the gain in body weight decreased immediately after the first injection of MTX and lasted until day 4. In Fig. 1, average changes in the body weight of control and MTX-treated rats are shown in time, relative to their individual weight at the onset of treatment at day -1 . Food consumption in the MTX-treated group was minimal at days 2 and 3 , when only $25 \%$ (i.e., $4 \mathrm{~g} / \mathrm{rat}$ ) of the intake of control rats was observed (not shown). By then, the MTX-treated rats had lost 2-10\% of their initial body weight. This weight loss was in part the result of dehydration because all MTX-treated rats developed diarrhea from day 2 until day 6. Recovery was seen from day 3 onward, accompanied by regain of appetite and increased gain in body weight compared with controls. During the experiment, no effect of MTX treatment on water intake was observed (not shown).

Proliferation. The effects of MTX on intestinal epithelial proliferation were analyzed through detection of incorporated BrdU, injected the day before the rats were killed. Because of the 24-h interval between injection and killing, BrdU-positive cells were not restricted to the crypts but were also found up to onethird of the length of the villi in all small intestinal regions of control rats due to migration of the cells (Fig. $2, A-C)$. In each small intestinal region, MTX completely inhibited epithelial proliferation, since BrdU injected $5 \mathrm{~h}$ after the final dose of MTX could not be detected in epithelial cells in any of the small intestinal

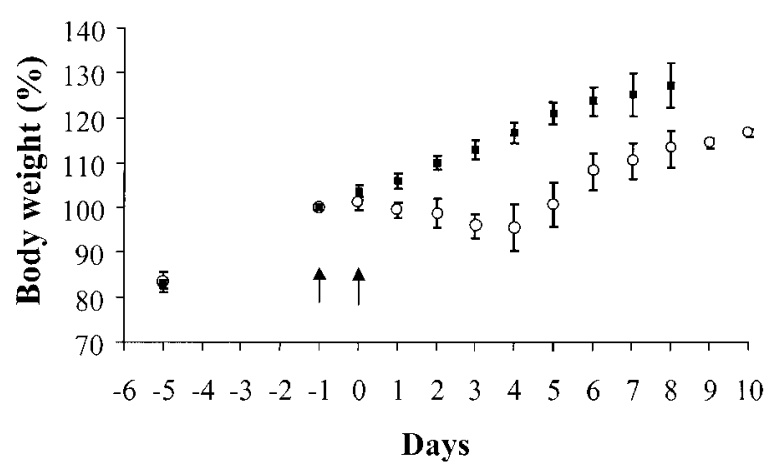

Fig. 1. Methotrexate (MTX)-induced effects on body weight. For each rat, body weight at the onset of treatment (day -1) was set arbi-

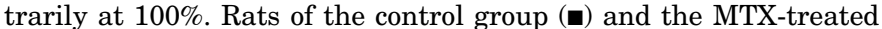
group (O) were injected intravenously using $\mathrm{NaCl}$ or MTX, respectively, at day -1 and day 0 (arrows). Individual changes in body weight relative to day -1 were recorded daily and averaged per group. Standard deviations are given in percentages of absolute weights. Rats of the MTX-treated group were killed sequentially until day 10. Control rats were killed at day 8 . 


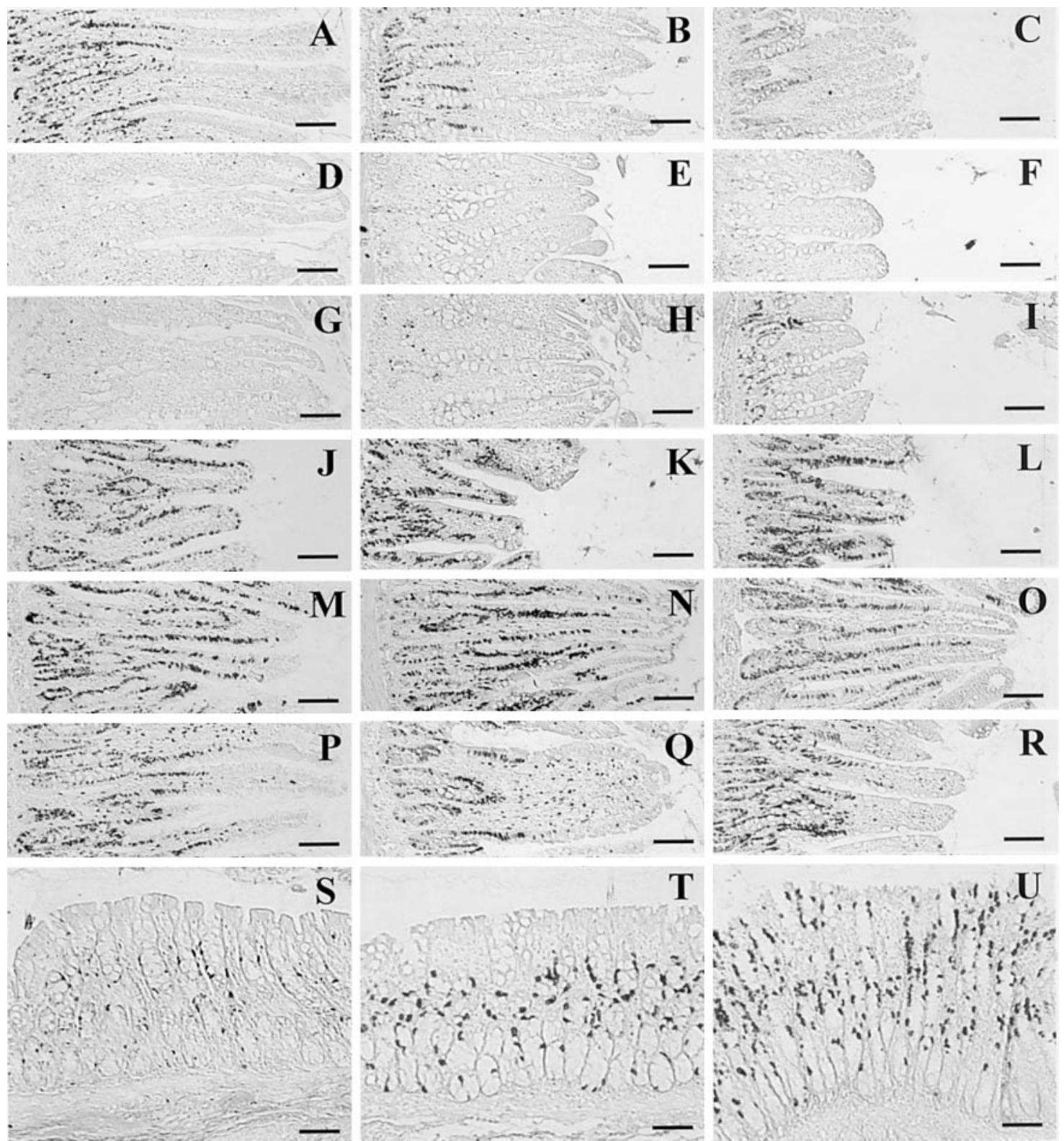

Fig. 2. Effects of MTX treatment on epithelial proliferation. Bromodeoxyuridine (BrdU) incorporation was detected immunohistochemically in the duodenum $(A, D, G, J, M, P)$, jejunum $(B, E, H, K, N, Q)$, ileum $(C, F, I, L, O, R)$ and colon $(S, T, U)$ of control and MTX-treated rats. In control rats, proliferation and migration resulted in BrdUpositive cells up to one-third of the villi in the small intestine $(A-C)$ and in the midcrypts of the colon $(S)$. After MTX treatment, proliferation was absent at day 1 along the entire small intestine $(D-F)$ and proliferation returned at first in the distal small intestine at day $2(G-I)$. Maximal proliferation was seen at day 4 along the entire small intestine $(J-L)$. Proliferation decreased gradually toward control patterns at day $6(M-O)$ and day $8(P-R)$. No decrease in BrdU staining was seen at day 1 in the colon $(T)$. Hyperproliferation was seen in the colon of some rats at day $6(U)$. Bars, $100 \mu \mathrm{m}$.

regions (Fig. 2, $D-F$ ). An induction of epithelial proliferation in the small intestine occurred between 24 and $48 \mathrm{~h}$ after the final dose of MTX in a region-dependent manner. A minor increase in the amount of BrdU staining was seen in duodenal crypts at day 2 (Fig. 2G). Slightly more BrdU-labeled cells were seen in the jejunum at day 2 (Fig. $2 H$ ), whereas the highest induction of proliferation was seen in the ileum (Fig. 2I). At day 3, BrdU-positive cells were abundantly covering the crypt epithelium and reached up to one-third of the length of the villi in all small intestinal regions (not shown). The numbers of BrdU-positive cells were further increased at day 4 in each region of the small intestine such that labeling was seen along the entire crypt-villus axes (Fig. 2, J-L). Between days 5 and 8, the numbers of BrdU-positive cells decreased toward control patterns in each of the small intestinal regions (Fig. 2, $M-R$ ).

Inhibition of proliferation was not seen in the colon in the days following MTX treatment because the 
BrdU-positive cells detected at days 1-3 in the midcrypt area remained very similar to controls (Fig. 2, $S$ and $T$ ). A remarkable induction of proliferation was observed in the colon between days 4 and 6 , when BrdU labeling occurred along the entire colonic crypt epithelium (Fig. 2U). However, this colonic hyperproliferation was not seen in each animal. After day 6 , the detection of BrdU in the colon of MTX-treated rats was comparable to the controls (not shown).

Morphology. Morphological damage and regeneration was analyzed on tissue sections through measurement of the length of the crypts and the villi in the small intestine and the colon. Due to time-dependent interanimal variations in the response to MTX administration, measurements from two consecutive days were pooled in the quantitative analysis of crypt depth and villus height (Fig. 3). Following the second MTX injection, the crypt depths were decreased in all small intestinal regions at days 1 and 2 compared with controls. This decrease was only significant in duodenum and jejunum, where crypt depths were $80 \%$ and $60 \%$ of controls, respectively. In all small intestinal regions, the crypts were significantly elongated on days 3 and 4 compared with days 1 and 2. The small intestinal crypts were further elongated on days 5 and 6 compared with controls or with days 3 and 4 . Between days 8 and 10, the crypt depth was comparable to controls in all small intestinal regions. In the colon, no significant changes occurred in the average crypt depth following MTX administration (data not shown). Nevertheless, it is worth mentioning that in some animals the colonic

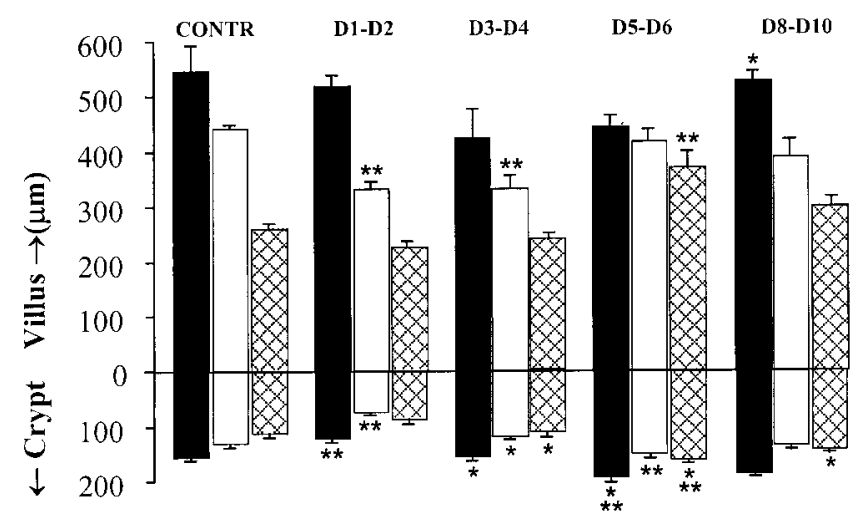

Fig. 3. Effects of MTX treatment on crypt depth and villus height in duodenum (filled bars), jejunum (open bars), and ileum (hatched bars). Mean crypt depth (downward) and mean villus height (upward) is shown per group per region. Crypt depth decreased in the small intestine until day 2 . In the proximal regions, this decrease was significant compared with controls $(P<0.05)$. During crypt regeneration on days 3 and 4 , they were enlarged compared with days 1 and 2 in all small intestinal regions $(P<0.05)$. Crypt elongation continued on days 5 and 6 , during which they even exceeded control values in duodenum and ileum. The length of the small intestinal villi decreased until day 4 after MTX treatment. Only in the jejunum was this decrease significant $(P<0.05)$. Small intestinal villi regenerated on days 5 and 6 , during which they were elongated in the ileum compared with controls. At days 8 and 10, the length of small intestinal crypts and villi returned within the range of controls. Values are means $\pm \mathrm{SE} . * P<0.05 \mathrm{vs}$. the previous group of the time course and $* * P<0.05$ vs. controls as analyzed using the Mann-Whitney $U$-test. crypts were enlarged to $150 \%$ of control length between days 4 and 6 , coinciding with increased BrdU incorporation in these animals, as shown in Fig. $2 U$.

The small intestinal villi progressively shortened between days 1 and 4 following MTX treatment compared with the length of the villi in controls. This villus atrophy was only significant in the jejunum $(75 \%$ of control values) and less pronounced in duodenum (80\% of control values) and ileum (85\% of control values). Between days 5 and 10, the villi regenerated to normal lengths in all small intestinal regions. However, during the regeneration phase at days 5 and 6 , the ileal villi temporarily exceeded the lengths of normal ileal villi ( $140 \%$ of controls).

Next to MTX-induced changes in small intestinal crypt and villus sizes, a morphological characteristic of epithelial damage became apparent when judged on HE-stained sections. At days 1 and 2, epithelial damage in the small intestine was characterized by a reduction in the number of crypts. In the remaining crypts, the epithelial cells appeared flattened. This damage was most severe in the duodenum (Fig. 4A). Toward the ileum, epithelial damage in the crypts was less pronounced (Fig. 4, $B$ and $C$ ), whereas in the colon morphological changes were absent (Fig. 4D). Epithelial cell damage was also observed on the villi at later stages in all small intestinal regions. At days 3 and 4, the villi showed reduced numbers of epithelial cells and the remaining cells were flattened, e.g., day 4 of the jejunum (Fig. 4, $E$ and $F$ ). Similar to the observed crypt damage, villus damage was also less severe toward the ileum (not shown). By days 5 and 6 , the villus epithelial cells appeared normal in number and in morphology in each small intestinal region (not shown).

MTX-induced apoptosis. In each of the small intestinal regions of controls, only an occasional crypt cell was TUNEL positive and most crypts were TUNEL negative (Fig. 5A). In MTX-treated rats, the number of TUNEL-positive cells was markedly increased at days 1 and 2 in the small intestinal crypts, but not on the villi (Fig. 5B). MTX-induced TUNEL-positive cells occurred in the crypts in all small intestinal regions, suggesting enhanced apoptosis at days 1 and 2 along the entire small intestine. The induction of apoptosis in small intestinal crypts at days 1 and 2 was further supported by the increased appearance of nuclear shrinkage and apoptotic bodies in serial HE-stained sections (not shown). In the colon, the number of TUNEL-positive cells was not altered in the crypts after MTX treatment compared with controls (not shown). Also, on serial sections stained with $\mathrm{HE}$, no signs of altered apoptosis were observed in the colon (not shown).

Epithelial gene expression during MTX-induced damage and regeneration. To localize differentiated enterocytes in the small intestine during epithelial damage, expression of AP and SI was detected immunohistochemically along the small intestine. In the jejunum of control small intestine, the brush borders of villus enterocytes were AP and SI positive (not shown). During maximal villus atrophy and epithelial restitu- 
Fig. 4. Hematoxylin and eosin-stained sections showing MTX-induced changes in epithelial morphology along the small intestine and colon. Crypt damage was seen in the small intestine and was characterized by loss of crypt cells and flattening of remaining epithelium (arrows). Crypt damage was most severe on day 2 in the duodenum $(A)$. Less severe damage was seen in the jejunum $(B)$ and the ileum $(C)$. No epithelial abnormalities were seen in the colon on day 2 $(D)$. During villus atrophy on days $2-4$ in all small intestinal regions, high numbers of epithelial cells were lost and the remaining cells appeared flattened, as shown in the jejunum on day $4(F)$ compared with controls $(E)$. v, Villus; s, serosal side. Bars, $50 \mu \mathrm{m}(A-D)$ and $10 \mu \mathrm{m}$ $(E-F)$.
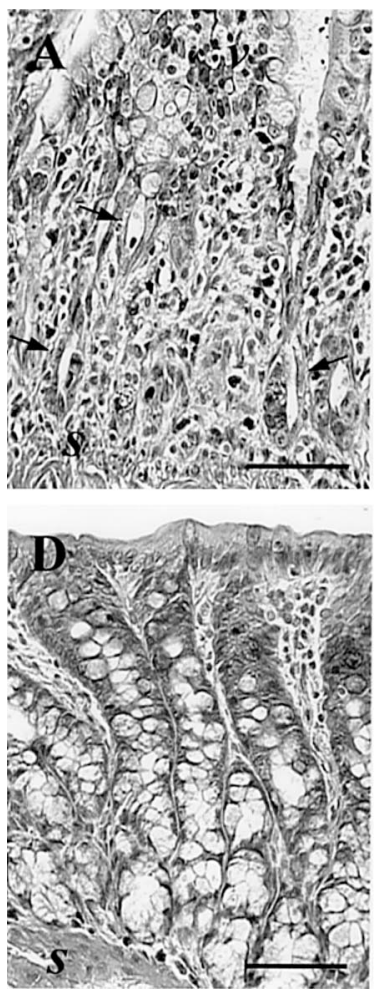
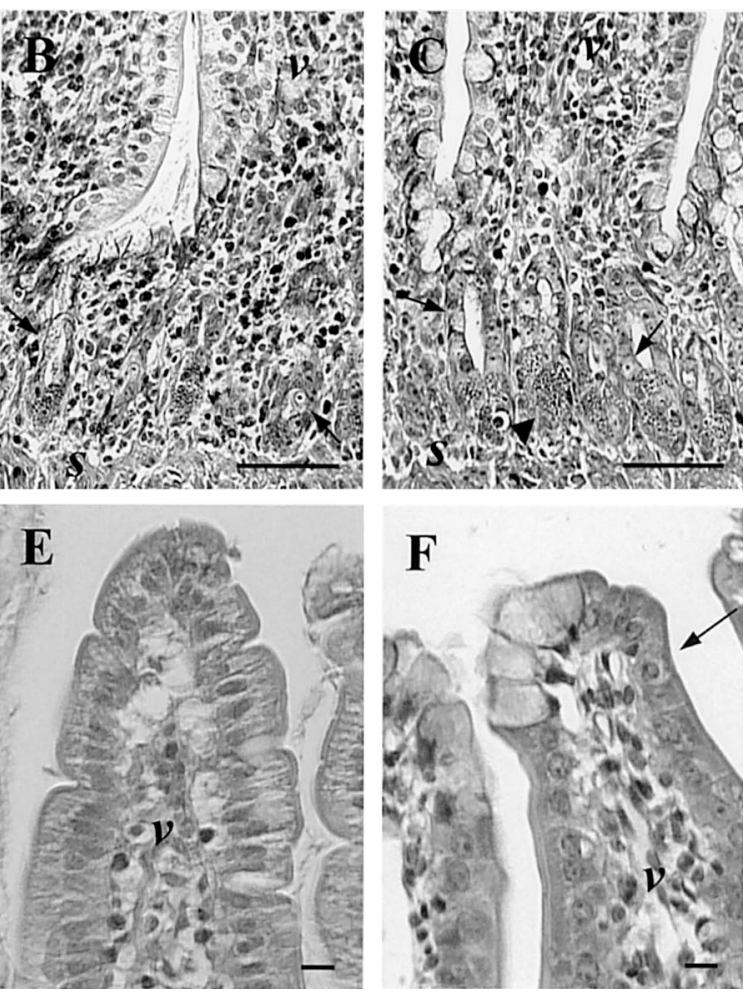

tion (days 3-4), villus enterocytes were reduced in number but were still AP and SI positive (e.g., Fig. 6G). To verify the functionality of AP during damage, its activity was localized on serial sections. The localization of AP activity in control jejunum was comparable to the immunohistochemical localization of the protein (Fig. 6A) and did not change during villus atrophy, as shown on day 4 (Fig. $6 B$ ). Similar results for AP and SI were also obtained for the duodenum and the ileum (not shown). Interestingly, in control jejunum, intracellular staining of the SI precursor is restricted to villus enterocytes localized close to the crypt-villus junction (not shown). The late onset of enterocyte differentiation during villus regeneration is reflected by the detection of SI in the Golgi region of enterocytes on villus tips on day 4 (Fig. $6 H$ ).

The presence and the functionality of goblet cells during MTX-induced damage and regeneration was analyzed in each small intestinal region using alcian blue staining of mucins in combination with the immunodetection of Muc2 and TFF3. In control small intestine, goblet cells increased in number toward the distal small intestine and were evenly distributed along the crypt-villus axis (Fig. 6A). At days 1 and 2, the number of goblet cells was decreased in the small intestinal crypts but not on the villi, as could be demonstrated using alcian blue staining and Muc2 or TFF3 immunostaining. This goblet cell depletion was most pronounced in the duodenum and was less pronounced toward the ileum (not shown). At days 3 and 4, goblet cells had returned in the crypts and became increasingly abundant on the tops of the villi in each region of the small intestine (e.g., Fig. 6B). These villus goblet cells, identified by alcian blue staining, were never positive for BrdU in double stainings (Fig. 6C). Apparently, the goblet cells but not the enterocytes were
Fig. 5. MTX-induced apoptosis in the small intestine of MTX-treated rats. Increased numbers of terminal transferase deoxyuridine nick-end labelingpositive cells (arrows) were seen in the crypts of the jejunum at days $1(B)$ and $2(C)$ compared with controls $(A)$. v, Villus; s, serosal side; *, crypts. Sections were counterstained with hematoxylin. Bars, $50 \mu \mathrm{m}$.
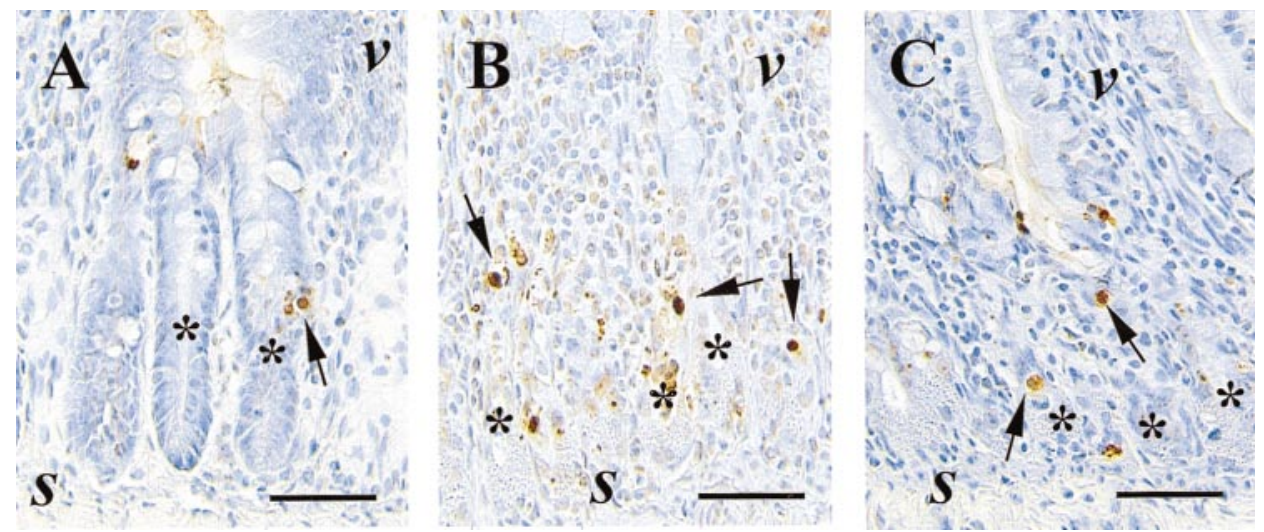

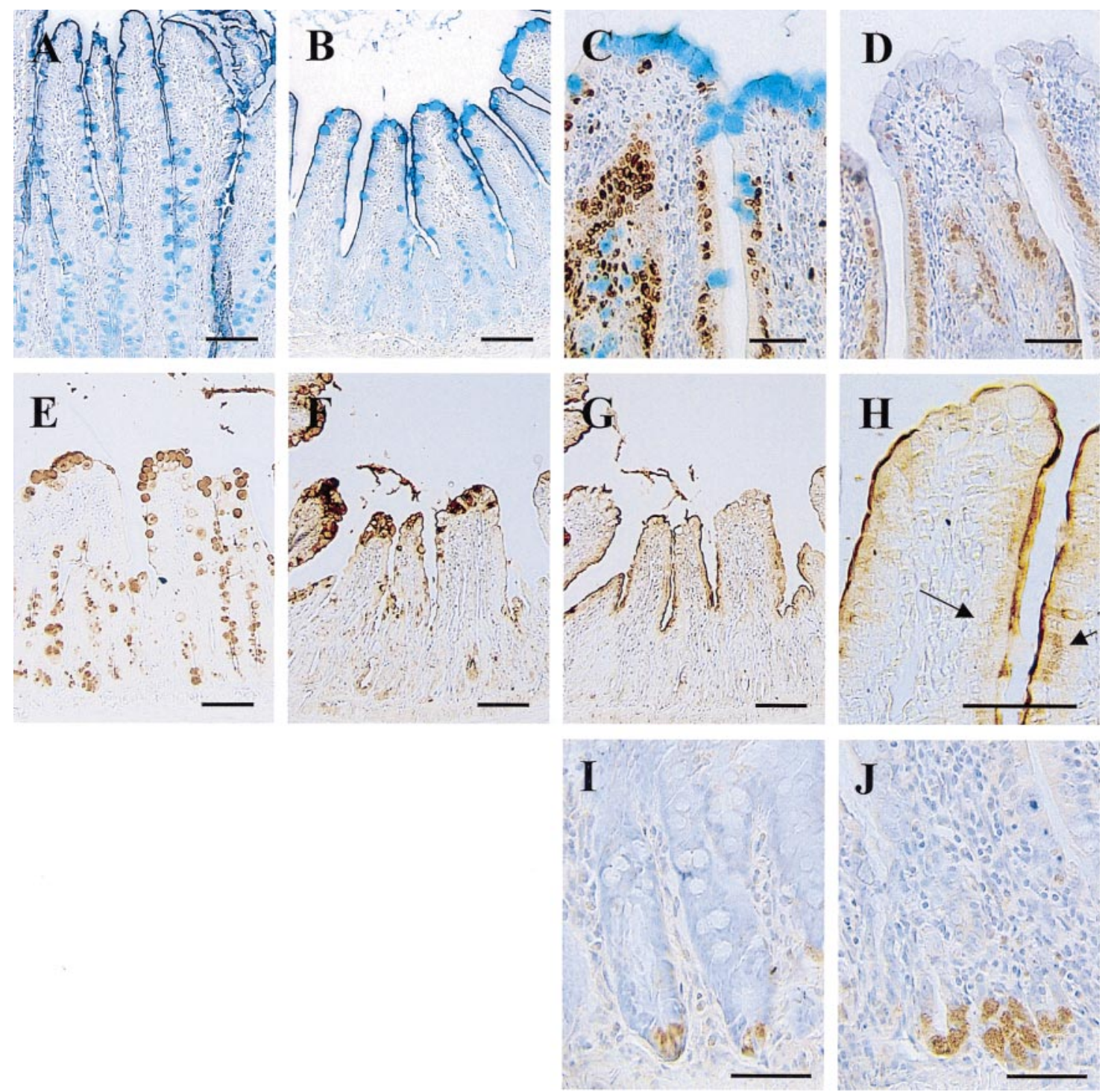

Fig. 6. Effects of MTX treatment on the (immuno-) histochemical detection of intestinal epithelial differentiation markers in rat jejunum and colon. $A$ and $B$ : alkaline phosphatase (AP) activity (dark blue) and alcian blue double staining in the jejunum of control $(A)$ and MTX-treated $(B)$ rats. AP activity remained detectable in the brush border of jejunal enterocytes during villus atrophy on day 4 after MTX treatment as in controls, despite severe cell loss. $C$ and $D$ : alcian blue and BrdU double staining of a jejunal section on day 4 after MTX treatment $(C)$ and a serial section stained for proliferating cell nuclear antigen (PCNA; D). During MTX-induced villus atrophy, goblet cells (in blue) accumulated at villus tips along the small intestine surrounded by BrdU- or PCNA-positive cells (in brown). $E$ and $F$ : goblet cell-specific expression of Muc2 $(E)$ and trefoil factor 3 (TFF3; $F$ ) at villus tips during MTX-induced villus atrophy on day 4 after MTX. $G$ and $H$ : sucrase-isomaltase (SI) expression in rat jejunum on $d a y$ 4 after MTX treatment. During MTX-induced villus atrophy, SI was decreased $(G)$ compared with controls (not shown). The intracellular staining of SI precursor detected high up the villus (arrows) indicates the late moment of enterocyte differentiation $(H) . I$ and $J$ : lysozyme immunostaining in Paneth cells of control jejunum $(I)$ and at day 2 after MTX $(J)$. Lysozyme expression was increased during crypt damage on days 1 and 2 after MTX treatment compared with controls. Bars, $50 \mu \mathrm{m}(C, D, H-J)$ and $100 \mu \mathrm{m}(A, B, E-G)$.

spared from extrusion, at least in the 24-h period following BrdU injection. This notion was confirmed using PCNA immunostaining as proliferation marker (Fig. 6D). The accumulating villus tip goblet cells were expressing both Muc2 (Fig. 6E) and TFF3 (Fig. 6F). To verify whether the increase in upper-villus goblet cells was statistically significant during villus atrophy, Muc2-positive cells were counted in three zones along the crypt-villus axis of control and MTX-treated jeju- num as a representative of the entire small intestine. In the jejunum of control animals, the goblet cells were almost evenly distributed along the crypt-villus axis (Table 1). From day 5 onward, the distribution pattern of goblet cells was normal in the small intestine (not shown).

Differentiated Paneth cells were identified in the base of the small intestinal crypts by their expression of lysozyme. Both in controls and in MTX-treated rats, 
Table 1. Numbers of goblet cells and Paneth cells in the jejunum of MTX-treated rats

\begin{tabular}{lrll}
\hline \hline & Control & Days 1 and 2 & Days 3 and 4 \\
\hline Goblet cells & & & \\
Villus tip & $11.00 \pm 1.70$ & $5.60 \pm 1.80^{*}$ & $9.50 \pm 2.50^{\dagger}$ \\
Villus base & $14.30 \pm 0.70$ & $7.70 \pm 1.98^{*}$ & $6.00 \pm 2.80^{\dagger}$ \\
Ratio, base:tip & $1.36 \pm 0.14$ & $1.64 \pm 0.43^{*}$ & $0.68 \pm 0.29^{* \dagger}$ \\
Paneth cells & $3.07 \pm 0.23$ & $3.52 \pm 0.38$ & $2.77 \pm 0.20$ \\
\hline
\end{tabular}

Average numbers of goblet cells and Paneth cells (mean \pm SD) were determined in well-orientated tissue sections by counting Muc2-positive and lysozyme-positive cells, respectively. For goblet cells, 2 zones along the villus axis were defined (see MATERIALS AND METHODS). Statistical analysis was performed using the Mann-Whitney $U$-test. ${ }^{*} P<0.05$ vs. control; ${ }^{\dagger} P<0.05$ vs. days 1 and 2 .

a gradual increase in the number of Paneth cells per crypt was seen from the duodenum toward the ileum (not shown). At days 1 and 2 after MTX treatment, the amount of lysozyme immunostaining was strongly increased in each of the small intestinal regions compared with controls, with a maximum at day 2 (Fig. 6, $I$ and $J$ ). During regeneration of the small intestinal crypts, from days 3 to 4 , lysozyme immunostaining returned to control levels (not shown).

In the colon, no changes were observed in the amount and distribution of either alcian blue-, Muc2-, or TFF3-positive goblet cells following MTX treatment (not shown). Differentiated colonic enterocytes were stained by their expression of CAI, a bicarbonate-producing enzyme present in the cytosol of colonic surface enterocytes. MTX treatment did not affect colonic CAI expression, except for some animals in which, during the late regeneration phase on days 6 and 8 , fewer CAI-positive cells were found in the colonic surface epithelium. This decrease may have been related to colonic hyperproliferation mentioned in Proliferation (not shown).

Sparing of goblet cells and Paneth cells. To investigate whether the observed accumulation of goblet cells at villus tips was statistically significant, Muc2-positive cells were counted along the crypt-villus axis, as described in MATERIALS AND METHODS. The numbers and distribution of Muc2-positive cells along the crypt-villus axis in the jejunum of controls and at days 1 and 2 and days 3 and 4 after MTX treatment are summarized in Table 1. In the two days following MTX treatment, the number of Muc2-positive cells was $\sim 50 \%$ reduced in both the upper half and the lower half of the villus (Table 1). In the lower half of the villi, the number of Muc2-positive cells was still decreased on days 3 and 4, although in contrast they had increased in villus tips to numbers that did not differ statistically from controls. This increase in Muc2-positive cells on villus tips on days 3 and 4 was statistically significant compared with days 1 and 2 and supported the observation of goblet cell sparing at villus tips during MTX-induced villus atrophy (Fig. 6).

The number of lysozyme-positive cells in the deeper crypts were counted on days 1-4 after MTX treatment and compared with controls to verify whether the num- ber of Paneth cells was affected by MTX (Table 1). However, the number of lysozyme-positive Paneth cells on days 1 and 2 and on days 3 and 4 were not statistically different from control values.

Quantitation of epithelial mRNA expression. Morphological damage was compared with alterations in levels of mRNA expression in the jejunum of MTXtreated rats using quantitative Northern blots. mRNA expression of CPSI and SI, markers for crypt and villus enterocytes, respectively, both decreased after MTX treatment (Fig. 7). CPSI decreased to $15 \%$ of control levels in 2 days and SI to $45 \%$ of control levels in 4 days. The mRNA expression of both enterocyte markers increased thereafter and reached control levels on days 5 and 6 . On days 8 and 10, the expression of CPSI and SI mRNA had further increased to $150 \%$ of control levels.

Goblet cell-specific mRNA expression was quantified using TFF3 and Muc2 as markers. In contrast to the enterocyte markers, TFF3 and Muc2 mRNA levels were maintained during damage on days 1 and 2 (Fig. 7). Lowest levels of TFF3 mRNA were seen during a period of extensive cell loss on days 3 and 4 (50\% of control), and lowest levels of Muc2 mRNA were seen during regeneration on days 5 and 6 (55\% of control). Both TFF3 and Muc2 mRNA levels gradually increased to control levels on days 8-10.

Lysozyme mRNA expression was quantified to investigate Paneth cell function. In agreement with the observed increase in lysozyme immunostaining, lysozyme mRNA was increased to $165 \%$ of control levels during damage on days 1 and 2 (Fig. 7). In the days thereafter, lysozyme mRNA expression was not statistically different from controls, except for a second phase of induction occurring on days 8-10.

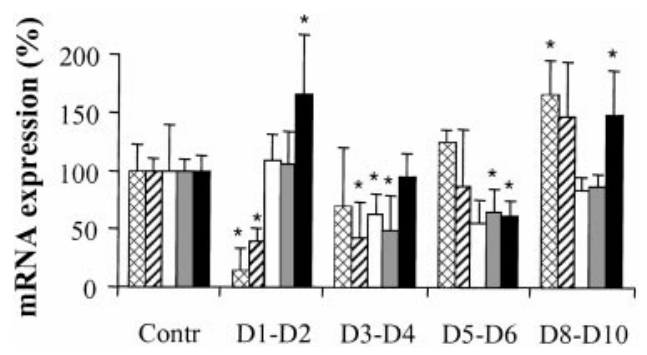

Fig. 7. Intestinal epithelial mRNA expression in normal and MTXtreated rats. mRNA expression was quantified on Northern blots and corrected for the amount of glyceraldehyde-3-phosphate dehydrogenase mRNA. Expression of carbamoyl phosphate synthase I (crosshatched bars) and SI (hatched bars) mRNA through enterocytes decreased during damage and gradually increased during and after regeneration to levels higher than control on days 8 and 10. Expression of goblet cell-specific Muc2 (gray bars) and TFF3 (open bars) mRNA remained stable during crypt damage (days 1 and 2) but were temporarily decreased during extensive cell loss on the villi (days 3 and 4). Expression of both markers returned to normal over the next few days. Lysozyme mRNA expression (filled bars) as marker for Paneth cell differentiation was increased during crypt damage and during late regeneration. ${ }^{*} P<0.05$ vs. control by Mann-Whitney $U$-test. 


\section{DISCUSSION}

The use of rats in this study allowed us to identify regional variation in intestinal epithelial sensitivity toward MTX and to gain insight into mechanisms of intestinal epithelial damage and regeneration after exposure to cytotoxic concentrations of MTX. These regional differences were reflected by a decrease in the degree of MTX-induced crypt damage along the proximal-to-distal axis of the intestine.

Analysis of epithelial proliferation after two consecutive days of MTX administration, using BrdU incorporation as a marker, demonstrated an inhibition of DNA synthesis in the epithelium of the entire small intestine until at least $5 \mathrm{~h}$ after the final dose. Proliferation of the colonic epithelium, on the other hand, was not inhibited compared with controls. In the small intestine, proliferation recurred $\sim 24 \mathrm{~h}$ after the final dose of MTX. This induction of proliferation was strongest in the ileum and decreased toward the duodenum. In vivo and in vitro proliferation studies in both human and rat have demonstrated a gradient of crypt cell production rates along the duodenal-colonic axis. In rat small intestine, the crypt cell production rate (cells $\cdot \operatorname{crypt}^{-1} \cdot \mathrm{h}^{-1}$ ) decreased from 28 in the duodenum to 12 in the ileum, whereas in the colon a decrease from 7.7 to 3 has been reported from proximal to distal $(1,6)$. The gradual decrease in crypt cell production rates from the duodenum toward the colon could explain the different sensitivity toward MTX.

Together with an inhibition of cell proliferation, MTX also induced apoptosis on days 1 and 2 in the proliferative region of small intestinal crypts, as judged by morphological criteria and TUNEL staining. In contrast, MTX-induced cell death was not observed in the colon. It is possible that the difference in apoptosis between small and large intestine is the result of regional differences in cell cycle control mechanisms following cellular damage, for instance, through the lower expression of p53 and the higher expression of $b c l-2$ in the colon compared with the small intestine (16). On the other hand, the colonic resistance to MTXinduced cell death could be directly related to the relative slow cell turnover in the colon, leading to only minor damage and less induction of cell death.

Morphological damage in the crypts of the small intestine paralleled increased apoptosis on days 1 and 2 . The observed flattening of the remaining crypt cells most likely represents epithelial restitution, which prevents breaches in the epithelial barrier. During crypt damage, CPSI mRNA expression was sixfold decreased in the jejunum, indicating the loss of crypt enterocyte function. Regeneration of the atrophic crypts started on day 3 in the small intestine via hyperproliferation and hyperplasia.

During MTX-induced villus atrophy (days 3 and 4), epithelial restitution was observed on the villi. Furthermore, SI mRNA expression was threefold decreased in the jejunum and paralleled the loss of villus enterocytes. It was previously shown that during MTXinduced villus atrophy, activities of the brush border glycohydrolytic enzymes SI and lactase are strongly downregulated in the small intestine (23). The absence of glycohydrolases indicates enterocyte malfunctioning with regard to the degradation and absorption of nutrients during villus atrophy. In contrast to the glycohydrolases, no decrease in the enterocytic expression of AP protein and enzyme activity was found during villus atrophy. Poelstra et al. (21) have indicated a role for AP in host defense against endotoxins. It is possible that expression of this protein is selectively maintained after MTX treatment to assist in the maintenance of the epithelial integrity. Especially during epithelial damage, as induced by MTX, epithelial defense is of vital importance for survival of the animal.

During regeneration, the lengths of the villi returned to normal in duodenum and jejunum. However, villus elongation continued in the ileum, and on days 5 and 6 abnormally long villi $(370 \mu \mathrm{m})$ were seen compared with controls $(260 \mu \mathrm{m})$. It is not clear why hyperplasia in the distal small intestine is more extensive than in the proximal small intestine. Also, in other studies describing the regeneration of intestinal villi following small bowel resection, increased villus lengths have been reported (15). It is possible that the feedback mechanisms involved in balanced cell proliferation are less controlled in the ileum compared with the proximal regions of the small intestine, because during regeneration not only the villi but also the crypts were $>40 \%$ longer compared with controls.

As in other studies (30), MTX induced a mild goblet cell depletion in the small intestine during a 4-day period of epithelial damage. Immunohistochemistry demonstrated that the MTX-induced goblet cell depletion mainly affected the villus base, whereas a remarkable sparing of goblet cells was observed at villus tips during the days of maximal villus atrophy (days 3 and 4). Furthermore, these accumulating cells were not labeled with BrdU and were PCNA negative. Apparently, these cells were spared from extrusion via an adaptive mechanism. Selective sparing of goblet cells could very well serve a protective function in epithelial defense via their secretion of mucins and trefoil factors $(14,25,27)$. Indeed, during early damage (days 1 and 2), small intestinal mRNA levels of Muc2 and TFF3 remained unchanged, although the number of goblet cells was 50\% reduced along the villi. Only during severe villus atrophy, when the amount of epithelial cells was minimal, was the expression of Muc2 and TFF3 mRNA decreased. Apparently, goblet cell function is very important during MTX-induced damage and therefore maintained. Another cell type with a pertinent role in epithelial defense is the Paneth cell. During crypt damage, it expresses $160 \%$ of normal lysozyme mRNA levels and shows increased intensity of lysozyme immunostaining. The phenomenon of Paneth cell hyperplasia through MTX treatment was described morphologically in electron microscopy studies (10). It is most likely that the slowly renewing Paneth cells not only have an advantage over the shorter-lived cell types in survival but that, importantly, the increased amounts of lysozyme expression 
indicate an increase in secretory capacity that very likely contributes to protection of the damaged mucosa.

In the colon, no significant changes could be observed in epithelial morphology and differentiation during the time course studied. The hyperproliferation and crypt elongation observed in some animals between days 4 and 6 may have been secondary effects in the response to MTX. For example, enhanced small intestinal repair mechanisms could lead to an increase in the amount of inductive signals, such as carbohydrates, fatty acids, bile salts, growth factors, and trefoil factors, in the intestinal lumen that could potentially stimulate proliferation in the downstream compartments.

In summary, MTX was shown to inhibit intestinal epithelial proliferation in the small intestine but not in the colon. Subsequent crypt damage was most severe in the duodenum, but regeneration through hyperproliferation was strongest in the distal small intestine. Interestingly, although severe damage could be induced in the small intestine by using MTX, Paneth cells, goblet cells, and enterocytes were all able to contribute to epithelial defense mechanisms. First, a direct increase of antimicrobial lysozyme expression was seen in Paneth cells, which remained high until crypt integrity was fully restored. Second, the goblet cells remained functional during early damage, were selectively spared from undergoing cell death, and accumulated at villus tips during villus atrophy, allowing secretion of the protective mucin and trefoil factor molecules. Third, despite the MTX-induced reduction in enterocyte numbers and SI and CPSI gene expression during villus atrophy, the putative detoxifier of endotoxins AP remained actively expressed in the small intestinal brush border. To gain further insight into regulatory mechanisms of epithelial defense and enterocyte function during MTX-induced damage and regeneration, additional studies are needed that will allow a quantitative analysis of cell type-specific gene expression. This insight is necessary to develop further clinically relevant strategies to protect cancer patients from intestinal damage during chemotherapy.

We thank Prof. D. K. Podolsky for kindly providing the rat TFF3 probe and the anti-Muc2 and anti-rat TFF3 antibodies. We thank Dr. R. Charles for providing both the anti-rat CPSI antibody and the rat CPSI probe. The anti-rat-SI antibody was a kind gift from Dr. H. P. Hauri. We thank Dr. W. S. Sly for providing the anti-rat CAI antibody. We also thank Dr. S. Krasinski, who kindly gave us the anti-rat SI probe and Dr. J. Power for his donation of the rat lysozyme probe.

This work was supported by Numico BV, Zoetermeer, The Netherlands.

\section{REFERENCES}

1. Al-Nafussi AI and Wright NA. The effect of epidermal growth factor (EGF) on cell proliferation of the gastrointestinal mucosa in rodents. Virchows Arch B Cell Pathol Incl Mol Pathol 40: 63-69, 1982.

2. Cheng H. Origin, differentiation and renewal of the four main epithelial cell types in the mouse small intestine. IV. Paneth cells. Am J Anat 141: 521-535, 1974.

3. Cheng $\mathbf{H}$ and Leblond CP. Origin, differentiation and renewal of the four main epithelial cell types in the mouse small intestine. III. Entero-endocrine cells. Am J Anat 141: 503-519, 1974.
4. Farrell CL, Bready JV, Rex KL, Chen JN, DiPalma CR, Whitcomb KL, Yin S, Hill DC, Wiemann B, Starnes CO, Havill AM, Lu ZN, Aukerman SL, Pierce GF, Thomason A, Potten CS, Ulich TR, and Lacey DL. Keratinocyte growth factor protects mice from chemotherapy and radiation-induced gastrointestinal injury and mortality. Cancer Res 58: 933-939, 1998.

5. Gaasbeek Janzen JW, Lamers WH, Moorman AF, de Graaf A, Los JA, and Charles R. Immunohistochemical localization of carbamoyl-phosphate synthetase (ammonia) in adult rat liver; evidence for a heterogeneous distribution. J Histochem Cytochem 32: 557-564, 1984.

6. Hall C, Youngs D, and Keighley MR. Crypt cell production rates at various sites around the colon in Wistar rats and humans. Gut 33: 1528-1531, 1992.

7. Hall PA, Coates PJ, Ansari B, and Hopwood D. Regulation of cell number in the mammalian gastrointestinal tract: the importance of apoptosis. J Cell Sci 107: 3569-3577, 1994.

8. Howarth GS, Cool JC, Bourne AJ, Ballard FJ, and Read LC. Insulin-like growth factor-I (IGF-I) stimulates regrowth of the damaged intestine in rats, when administered following, but not concurrent with, methotrexate. Growth Factors 15: 279-292, 1998.

9. Howarth GS, Francis GL, Cool JC, Xu X, Byard RW, and Read LC. Milk growth factors enriched from cheese whey ameliorate intestinal damage by methotrexate when administered orally to rats. J Nutr 126: 2519-2530, 1996.

10. Jeynes BJ and Altmann GG. Light and scanning electron microscopic observations of the effects of sublethal doses of methotrexate on the rat small intestine. Anat Rec 191: 1-17, 1978.

11. Kanauchi O, Mitsuyama K, Saiki T, Agata K, Nakamura T, and Iwanaga T. Preventive effects of germinated barley foodstuff on methotrexate- induced enteritis in rats. Int J Mol Med 1: 961-966, 1998.

12. Krasinski SD, Estrada G, Yeh KY, Yeh M, Traber PG, Rings EH, Buller HA, Verhave M, Montgomery RK, and Grand RJ. Transcriptional regulation of intestinal hydrolase biosynthesis during postnatal development in rats. Am J Physiol Gastrointest Liver Physiol 267: G584-G594, 1994.

13. Logvinova AV, Foehr MW, Pemberton PA, Khazalpour KM, Funk-Archuleta MA, Bathurst IC, and Tomei LD. Soy-derived antiapoptotic fractions protect gastrointestinal epithelium from damage caused by methotrexate treatment in the rat. Nutr Cancer 33: 33-39, 1999.

14. Mashimo H, Wu DC, Podolsky DK, and Fishman MC. Impaired defense of intestinal mucosa in mice lacking intestinal trefoil factor. Science 274: 262-265, 1996.

15. McDermott FT and Roudnew B. Ileal epithelial cell migration after $40 \%$ small-bowel resection. Autoradiographic studies in the rat. Am J Dig Dis 22: 637-640, 1977.

16. Merritt AJ, Potten CS, Kemp CJ, Hickman JA, Balmain A, Lane DP, and Hall PA. The role of p53 in spontaneous and radiation-induced apoptosis in the gastrointestinal tract of normal and p53-deficient mice. Cancer Res 54: 614-617, 1994.

17. Moss SF, Calam J, Agarwal B, Wang S, and Holt PR. Induction of gastric epithelial apoptosis by Helicobacter pylori. Gut 38: 498-501, 1996.

18. Nagai Y, Horie T, and Awazu S. Vitamin A, a useful biochemical modulator capable of preventing intestinal damage during methotrexate treatment. Pharmacol Toxicol 73: 69-74, 1993.

19. Nyunoya H, Broglie KE, Widgren EE, and Lusty CJ. Characterization and derivation of the gene coding for mitochondrial carbamyl phosphate synthetase I of rat. J Biol Chem 260: 93469356, 1985.

20. Pinkerton CR, Cameron CH, Sloan JM, Glasgow JF, and Gwevava NJ. Jejunal crypt cell abnormalities associated with methotrexate treatment in children with acute lymphoblastic leukaemia. J Clin Pathol 35: 1272-1277, 1982.

21. Poelstra K, Bakker WW, Klok PA, Kamps JA, Hardonk MJ, and Meijer DK. Dephosphorylation of endotoxin by alkaline phosphatase in vivo. Am J Pathol 151: 1163-1169, 1997. 
22. Suemori S, Lynch-Devaney K, and Podolsky DK. Identification and characterization of rat intestinal trefoil factor: tissueand cell-specific member of the trefoil protein family. Proc Natl Acad Sci USA 88: 11017-11021, 1991.

23. Taminiau JA, Gall DG, and Hamilton JR. Response of the rat small-intestine epithelium to methotrexate. Gut 21: 486$492,1980$.

24. Tanaka H, Miyamoto KI, Morita K, Haga H, Segawa H, Shiraga T, Fujioka A, Kouda T, Taketani Y, Hisano S, Fukui Y, Kitagawa K, and Takeda E. Regulation of the PepT1 peptide transporter in the rat small intestine in response to 5-fluorouracil-induced injury. Gastroenterology 114: 714-723, 1998.

25. Tytgat KM, Bovelander FJ, Opdam FJ, Einerhand AW, Buller HA, and Dekker J. Biosynthesis of rat MUC2 in colon and its analogy with human MUC2. Biochem J 309: 221-229, 1995.

26. Tytgat KM, Klomp LW, Bovelander FJ, Opdam FJ, Van der Wurff A, Einerhand AW, Buller HA, Strous GJ, and Dekker J. Preparation of anti-mucin polypeptide antisera to study mucin biosynthesis. Anal Biochem 226: 331-341, 1995.

27. Van Klinken BJ, Dekker J, Buller HA, and Einerhand AW. Mucin gene structure and expression: protection vs. adhesion. Am J Physiol Gastrointest Liver Physiol 269: G613-G627, 1995.
28. Walters JR, Howard A, Rumble HE, Prathalingam SR, Shaw-Smith CJ, and Legon S. Differences in expression of homeobox transcription factors in proximal and distal human small intestine. Gastroenterology 113: 472-477, 1997.

29. Westcarr S, Farshori P, Wyche J, and Anderson WA. Apoptosis and differentiation in the crypt-villus unit of the rat small intestine. J Submicrosc Cytol Pathol 31: 15-30, 1999.

30. Xian CJ, Howarth GS, Mardell CE, Cool JC, Familari M, Read LC, and Giraud AS. Temporal changes in TFF3 expression and jejunal morphology during methotrexate-induced damage and repair. Am J Physiol Gastrointest Liver Physiol 277: G785-G795, 1999.

31. Xu G, Huan LJ, Khatri IA, Wang D, Bennick A, Fahim RE, Forstner GG, and Forstner JF. cDNA for the carboxyl-terminal region of a rat intestinal mucin-like peptide. J Biol Chem 267: 5401-5407, 1992.

32. Yeh KY, Yeh M, and Holt PR. Thyroxine and cortisone cooperate to modulate postnatal intestinal enzyme differentiation in the rat. Am J Physiol Gastrointest Liver Physiol 260: G371G378, 1991.

33. Yogalingam G, Doyle IR, and Power JH. Expression and distribution of surfactant proteins and lysozyme after prolonged hyperpnea. Am J Physiol Lung Cell Mol Physiol 270: L320L330, 1996.

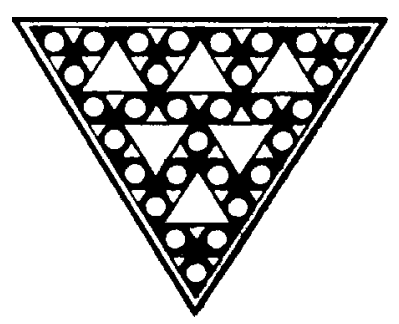

